\title{
On the Factors That Affect the Mode of Cyclization of $\alpha$-Silyl Radicals: A Method for Angular Hydroxymethylation by the 5-Exo Cyclization
}

\author{
Masato Koreeda* and Daniel C. Visger \\ Department of Chemistry, The University of Michigan, Ann Arbor, Michigan 48109-1055
}

\begin{abstract}
The steric and stereochemical factors which favor the 5-exo cyclization of a 5-substituted 2-sila-5hexen-1-yl radical have been probed. This radical cyclization approach constitutes a highly efficient and convenient means for the stereospecfic introduction of an angular hydroxymethyl group.
\end{abstract}

The use of 2-sila-5-hexen-1-yl radicals as a means of introducing a hydroxymethyl equivalent to a stereogenic olefinic center has attracted considerable attention in recent years. ${ }^{1-3}$ Earlier studies concentrated mostly on the 5-exo mode of cyclization of the $\alpha$-silyl radicals of endocyclic allylic alcohols. ${ }^{1-3}$ During our study on the synthesis of 22-hydroxylated steroids, the aspect of 5-exo vs. 6-endo mode of cyclization of these radicals was examined for the $\alpha$-silyl radical derivatives of exocyclic allylic alcohols. ${ }^{4}$ In line with the enhanced propensity for the 6-endo cyclization of acyclic 2-sila-5-hexen-1-yl radicals, 2,5 even the pseudoaxially oriented 16ß-derivative 1, generated from its bromo-precursor by tributyltin hydride and AIBN, was found to undergo predominantly the 6-endo mode of cyclization (path a), providing siloxane 2 . Interestingly, its $Z$ olefin isomer 3, where the 6-endo cyclization is expected to be impeded due to the steric interaction with the 21-methyl hydrogens, produced only the reduction product, i.e., the trimethylsilyl ether of the 16ß-hydroxy17Z-ethylidene. ${ }^{4}$ However, the role of the 18 -methyl hydrogens in the course of these radical cyclizations
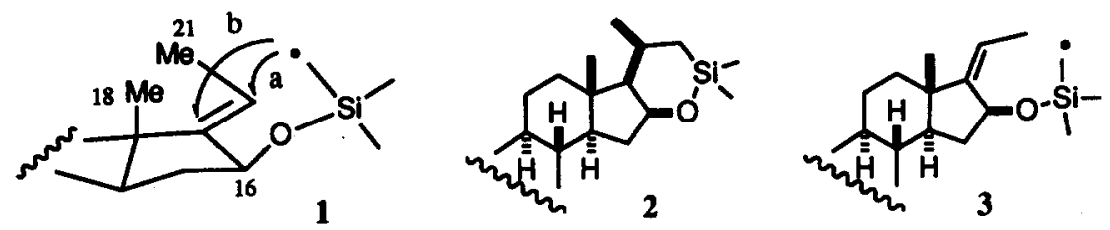

remained to be elucidated. To this end, (bromomethyl)dimethylsilyl ethers of several conformationally well defined model systems 7a - 12a have been synthesized and the steric and stereochemical factors which control the mode of cyclization of the corresponding $\alpha$-silyl radicals have been probed. In this communication, we report, in addition to the results from these studies, an example of clean 5-exo cyclization of a 5-substituted 2sila-3-oxa-5-hexen-1-yl radical, which represents a novel means for the introduction of an angular hydroxymethyl group (see Scheme 1).

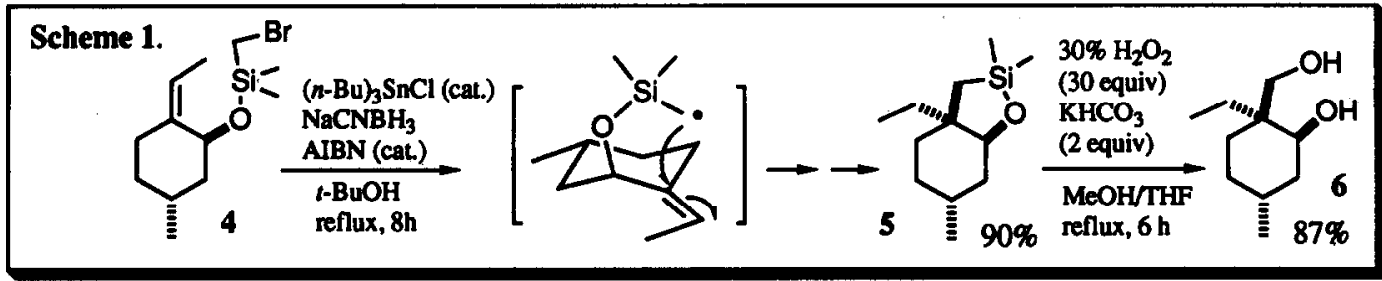




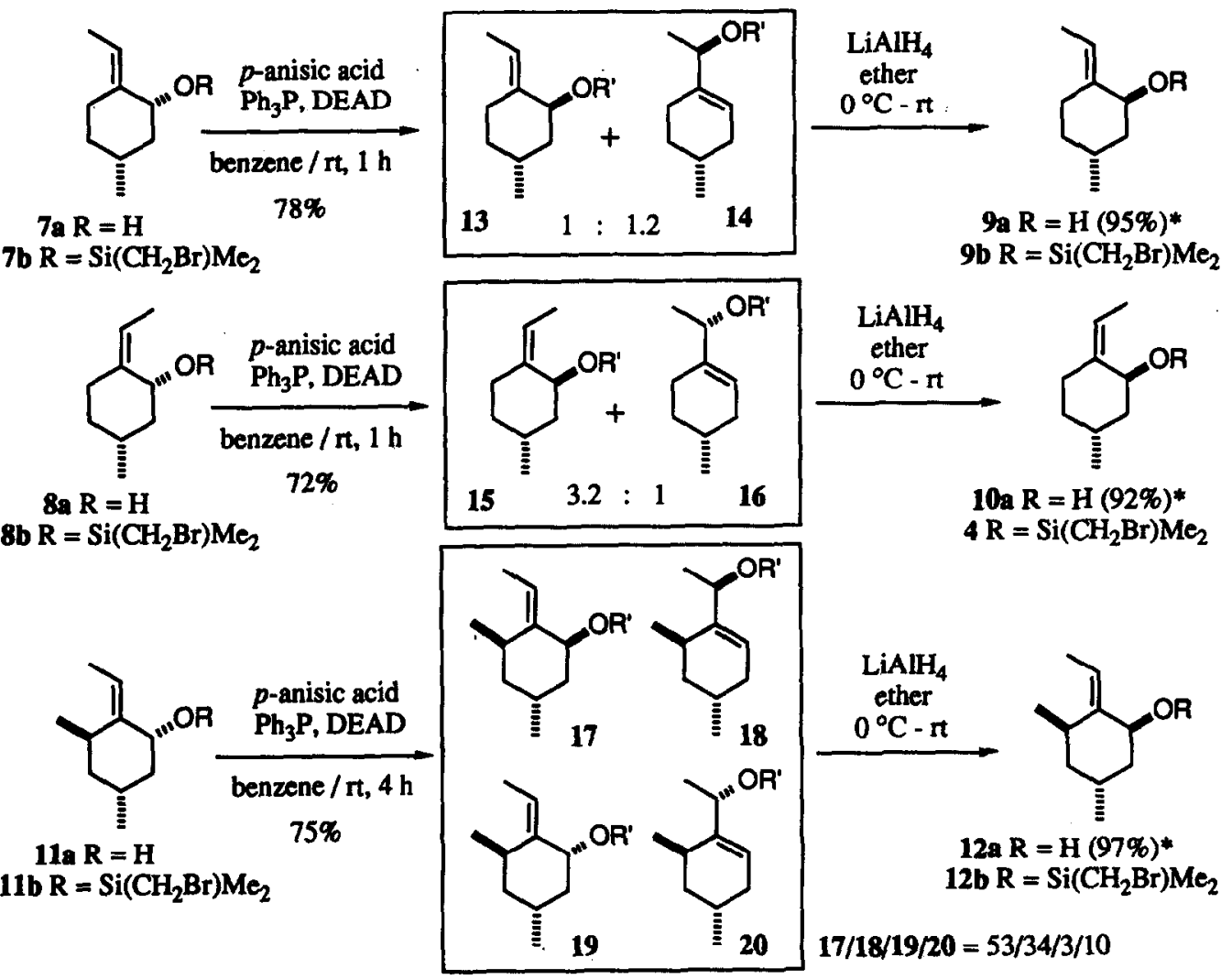

Figure 1. Synthesis of Allylic Alcohols. Note: R' = p-methoxybenzoyl. *Yields of chromatographically purified products calculated on the basis of the adjusted fraction of their corresponding $p$-methoxybenzoyl precursors.

The $\beta$-allylic alcohols 9a, 10a, and $12 \mathrm{a}$ were prepared from their $\alpha$-hydroxy isomers 7a, $8 \mathrm{aa}, 6$ and $11 \mathrm{a},{ }^{7}$ respectively by the Mitsunobu reaction followed by LAH reduction as summarized in Figure 1 . The inversion of the hydroxy configuration employed the protocol introduced by Martin, ${ }^{8}$ producing the desired esters along with the products from competing reactions such as that through the $\mathrm{S}_{\mathrm{N}} 2^{\prime}$ pathway. Particularly, the Mitsunobu reaction of 11 a was found to be highly problematic in that it produced all four conceivable products in which the desired ester with the inverted configuration was the major. It should be noted that the stereochemistry of the p-methoxybenzoate derivatives 14, 16, 18, and 20 remains to be unequivocally established. Each of the resulting ester mixtures from the Mitsunobu reaction was treated with $\mathrm{LiAlH}_{4}$ and the desired hydroxy-inverted allylic alcohol was isolated by flash column chromatography with silica gel. The (bromomethyl)dimethylsilyl ether derivatives of $7 \mathrm{a}, 9 \mathrm{a}, 10 \mathrm{a}, 11 \mathrm{a}$, and $12 \mathrm{a}$ were obtained smoothly in over $90 \%$ yield with $\left(\mathrm{BrCH}_{2}\right) \mathrm{Me2} \mathrm{SiCl}\left(1.05\right.$ equiv)/Et ${ }_{3} \mathrm{~N}\left(2.0\right.$ equiv) in $\mathrm{CH}_{2} \mathrm{Cl}_{2}\left(0^{\circ} \mathrm{C}-\mathrm{rt}, 2 \mathrm{~h}\right)$. In contrast, the derivatization of the sterically congested allylic alcohol $11 \mathrm{a}$ required the generation of the alkoxide with 1.0 equiv of $n$-BuLi in THF at $-78^{\circ} \mathrm{C}$ prior to the addition of the silylating agent, providing $8 \mathrm{~b}$ in $92 \%$ yield. 
$\alpha$-Silyl radicals were generated from these six $\alpha$-bromosilyl ethers by either a stoicheometric or catalytic tributyltin hydride method (Method A or B, respectively, in Table 1) and the results of their cyclization are shown in Scheme 1 and Table 1. While the catalytic tinhydride method developed by Stork ${ }^{1 b}$ is advantageous in the subsequent purification of these nonpolar siloxane products due to the reduced amount of the tin species utilized, equatorial silyl ethers $\mathbf{7 b}, \mathbf{8 b}$, and $\mathbf{1 1 b}$ required the use of a stoicheometric amount of tributyltin hydride. It is of interest to note that the radical cyclizations of these silyl ethers were sluggish and the yields of the siloxane products were lower than those from the axially oriented silyl ethers $9 \mathrm{~b}, 4$, and $12 \mathrm{~b}$. The stereostructures of these siloxanes were assigned primarily on the basis of the analysis of the spin-spin couplings involving hydrogens on the siloxane ring. ${ }^{4}$ In addition, the structure of 26 was further validated by the single crystal $x$-ray analysis of its diol derivative obtained through oxidation with $\mathrm{H}_{2} \mathrm{O}_{2} / \mathrm{KHCO}_{3}{ }^{9}$ in

Table 1. Results of $\alpha$-Silyl Radical Cyclization [note: $\mathrm{R}=\left(\mathrm{BrCH}_{2}\right) \mathrm{Me}_{2} \mathrm{Si}$ ]

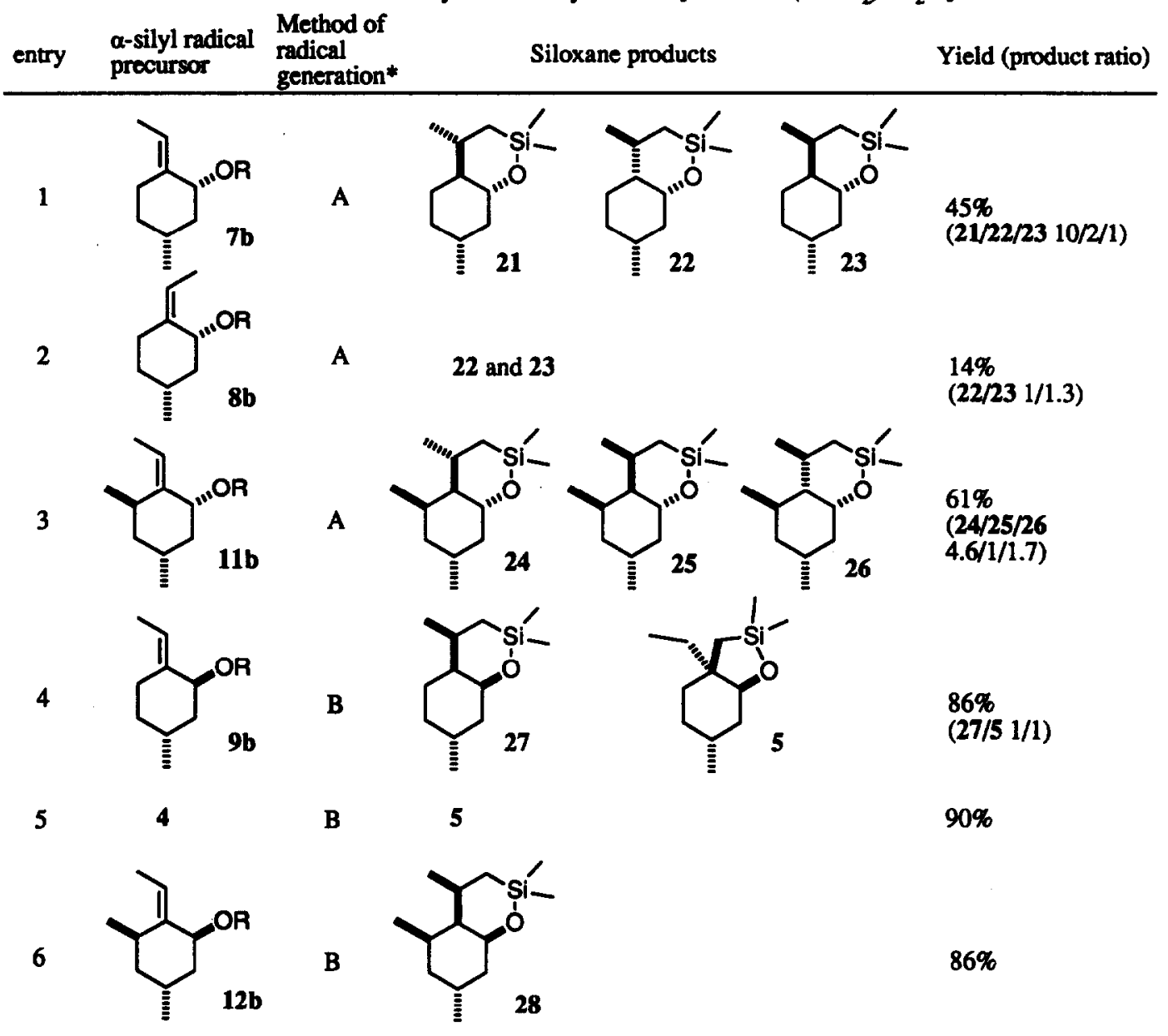

*Method A: ( $n$-Bu $)_{3}$ SnH (1.25 equiv) and AIBN ( 0.05 equiv) ( $12 \mathrm{~h}$-addition), benzene, reflux, $16 \mathrm{~h}$ Method B: $(n-\mathrm{Bu})_{3} \mathrm{SnCl}\left(0.05\right.$ equiv), $\mathrm{NaCNBH}_{3}$ (2.0 equiv), AIBN $(0.05$ equiv) $/ t$-BuOH, reflux, $8 \mathrm{~h}$ 
THF/MeOH. Details of these proton NMR and $x$-ray analyses will be described in our forthcoming full account.

The results shown in Table 1 are highly informative regarding the structural factors that favor the 5-exo cyclization of these radicals. Having the $\alpha$-silyloxy radicals oriented axially to the ethylidenecyclohexane ring kinetically enhances the propencity for the 5-exo cyclization relative to those oriented equatorially. Comparison of the cyclization of the radicals from isomeric $9 \mathrm{~b}$ and 4 is quite revealing as to the steric influence of the methyl group at the olefinic carbon onto which the radical can add. The presence of such a $Z$-methyl group impedes completely the 6-endo cyclization, yielding exclusively the product from the 5-exo cyclization (entry 5). However, the presence of a 1,3-diaxially oriented methyl group in the ring suppresses the 5-exo mode of cyclization as observed for the radical from $12 \mathrm{~b}$ (entry 6 ) and the steroidal radical 1 . It is evident that the reactions of the radicals from 4 and $12 b$ (see also Scheme 1) represent an optimum combination of these stereochemical and steric factors. It is of interest to note that a recent report by Lejeune and Lalleman 10 delineates an electronic factor in a similar system that enhances the propensity for the 5-exo $\alpha$-silyloxy radical cyclization. All of these 5- and 6-membered siloxanes can be readily oxidized in excellent yields to their respective diols with $\mathrm{H}_{2} \mathrm{O}_{2} / \mathrm{KHCO}_{3}$ in THF/MeOH (see, for example, 5 to 6 in Scheme 1). The preparation of these diols and their use in synthesis will be published as a separate article.

In conclusion, the steric and sterochemical factors which favor the 5-exo cyclization of a 5-substituted 2sila-5-hexen-1-yl radical have been probed. This radical cyclization approach constitutes a highly efficient and convenient means for the stereospecfic introduction of an angular hydroxymethyl group.

Acknowledgment. This work was supported by the National Institutes of Health (Grant No. DK30025).

\section{References}

1. (a) Stork, G.; Kahn, M. J. Am. Chem. Soc. 1985, 107, 500. (b) Stork, G.; Sher, P. M. Ibid. 1986, 108, 303. (c) Stork, G.; Sofia, M. J. Ibid. 1986, 108, 6826.

2. Nishiyama, H.; Kitajima, T.; Matsumoto, M. J. Org. Chem. 1984, 49, 2298.

3. Reviews: (a) Curran, D. P. Synthesis 1988, 417 and 489. (b) Jasperse, C. P.; Curran, D. P.; Fevig, T. L. Chem. Rev. 1991, 91, 1237.

4. (a) Koreeda, M.; George, I. A. J. Am. Chem. Soc. 1986, 108, 8098. (b) Idem Chem. Lett. 1990, 83.

5. (a) Wilt, J. W. J. Am. Chem. Soc. 1981, 103, 5251. (b) Idem. Tetrahedron 1985, 41, 3925. (c) Wilt, J. W.; Lusztyk, J.; Peeran, M.; Ingold, K. U. J. Am. Chem. Soc. 1988, 110, 281.

6. Koreeda, M.; Brown, L. J. Org. Chem. 1983, 48, 2122.

7. Koreeda, M.; You, Z. J. Org. Chem. 1989, 54, 5195.

8. Martin, S. F.; Dodge, J. A. Tetrahedron Lett. 1991, 32, 3017.

9. (a) Tamao, K.; Ishida, N.; Tanaka, T.; Kumada, M. Organometallics 1983, 2, 1694. (b) Tamao. K.; Ishida, N.; Kumada, M. J. Org. Chem. 1983, 48, 2120.

10. Lejeune, J.; Lallemand, J. Y. Tetrahedron Lett. 1992, 33, 2977. 